\title{
Atypical Femoral Fractures due to long-term bisphosphonates therapy
}

\author{
Ioan Mihai Japie*, Radu Rădulescu***, Adrian Bădilă ***, Ecaterina-Maria Japie***, Alexandru \\ Papuc*, Traian Ciobanu *, Adrian Dumitru****, Cătălin Cîrstoiu* ** \\ *Department of Orthopaedics and Traumatology, University Emergency Hospital, Bucharest, Romania \\ ** "Carol Davila" University of Medicine and Pharmacy, Bucharest, Romania \\ ***Department of Pediatric Orthopaedics, Marie Sklodowska Curie Children's Clinical Hospital, \\ Bucharest, Romania \\ $* * * *$ Department of Pathology, University Emergency Hospital, Bucharest, Romania
}

Correspondence to: Ioan-Mihai Japie, MD, PhD Student, Department of Orthopaedics and Traumatology, University Emergency Hospital, Bucharest, 169 Splaiul Independentei Street, Code 050098, District 5, Bucharest, Romania, Phone: +4021 31805 23, E-mail: japie.ioan.mihai@gmail.com

\begin{abstract}
Introduction. Bisphosphonates (BPs) represent the main therapy in patients with osteoporosis, although a long-term treatment can lead to atypical fractures.

Material and methods. We conducted a retrospective study between 2008 and 2017 and included 23 female patients with atypical femoral fractures (AFFs). The mean period of BPs therapy administration was 5.2 years. We included 7 subtrochanteric fractures and 18 femoral shaft fractures. Two of the total patients presented bilateral femoral fractures. 18 patients presented prodromal symptoms from 2 to 18 months before the diagnosis of fractures, all of them following low energy trauma. All the patients included in our study underwent surgery with intramedullary nail or gamma nail. The postoperative mean follow-up was 2 years. Results. Of all 23 female patients with a total of 25 fractures -10 underwent osteosynthesis with gamma nail and 15 underwent osteosynthesis with intramedullary nail. From the total number of patients: 13 patients achieved complete union, 6 presented delayed union and 3 non-union ( 2 intramedullary nail and one gamma nail), whereas in one patient treated previously with gamma nail we observed implant failure.

Conclusion. The treatment of AFFs after BPs therapy administration represents a challenge for orthopaedic surgeons regarding both surgical technique and postoperative follow-up.

Keywords: osteoporosis, bisphosphonates, atypical femoral fractures, gamma nail, intramedullary nail
\end{abstract}

\section{Introduction}

Osteoporosis represents a metabolic disease characterized by the reduction of mineral bone density and alteration of bone architecture [1] thus being a trigger factor for fractures. Several studies concluded that the incidence of osteoporosis is $50 \%$ in females and $25 \%$ in males [2]. As life expectancy increased over the years, so did the incidence of fractures in osteoporotic patients. Bisphosphonates (BPs) represent the main therapy in patients with osteoporosis. BPs decrease bone resorption by inhibiting osteoclasts [3], thus improving bone density and reducing the risk of fractures especially those localized in the spine and hip [4]. However, in 2005, Odvina described the reduction of bone turnover secondary to 
long-term treatment with alendronate, hence leading to atypical fractures [5].

\section{Materials and methods}

We conductedaretrospectivestudybetween 2008 and 2017 and included 23 female patients with atypical femoral fractures (AFFs) assessed with the American Society of Bone and Mineral Research (ASBMR) diagnostic criteria (Table 1). The mean age was 69 years old (extreme ages 57 and 81 years old respectively). The period of BPs therapy administration was between 2 to
7 years until a femoral fracture was produced (the mean period was 5.2 years). We included 7 subtrochanteric fractures and 18 femoral shaft fractures. Two of the total number of patients presented bilateral fractures. 18 patients presented prodromal symptoms from 2 to 18 months before the diagnosis of fractures, all of them following low energy trauma. All the patients included in our study underwent surgery with intramedullary nail or gamma nail. The postoperative mean follow-up was 2 years.

Table 1. The 2014 AFFs diagnostic criteria as determined by the ASMBR task force statements [6]

Major features
The fracture is associated with minimal or no
trauma, as in a fall from a standing height or less
The fracture line originates at the lateral cortex
and is substantially transverse in its orientation,
although it may become oblique as it progresses
medially across the femur
Complete fractures extend through both cortices
and may be associated with a medial spike;
incomplete fractures involve only the lateral
cortex
The fracture is non-comminuted or minimally
comminuted
Localized periosteal or endosteal thickening of
the lateral cortex is present at the fracture site
("beaking" or "flaring")

\section{Results}

Results were evaluated postoperatively from a radiological point of view in two incidences (anterior-posterior and laterolateral) at 6 weeks, 3, 6, 9 and 12 months respectively.

Of all 23 female patients with a total of 25 fractures -15 underwent osteosynthesis with intramedullary nail (Fig. 1) and 10 underwent osteosynthesis with gamma nail (Fig. 2). Regarding the period of time needed for the consolidation of fracture we obtained the following results: 13 patients achieved

\section{Minor features}

Generalized increase in cortical thickness of the femoral diaphyses

Unilateral or bilateral prodromal symptoms such as dull or aching pain in the groin or thigh

Bilateral incomplete or complete femoral diaphysis fractures

Delayed fracture healing

To satisfy the case definition of AFF, the fracture must be located along the femoral diaphysis from just distal to the less trochanter to just proximal to the supracondylar flare

complete union, 6 presented delayed union and 3 non-union ( 2 intramedullary nail and one gamma nail), whereas in one patient treated previously with gamma nail we observed an implant failure. The mean period of union was 26 weeks in patients with complete union and 30 weeks when taking into consideration also the patients with delayed consolidation. Among the 6 patients with delayed consolidation 4 presented subtrochanteric fractures and 2 presented femoral shaft fractures.

In our study, we also practiced biopsy with subsequent histopathological examination from the fracture site (Fig. 3 and 4 ). 

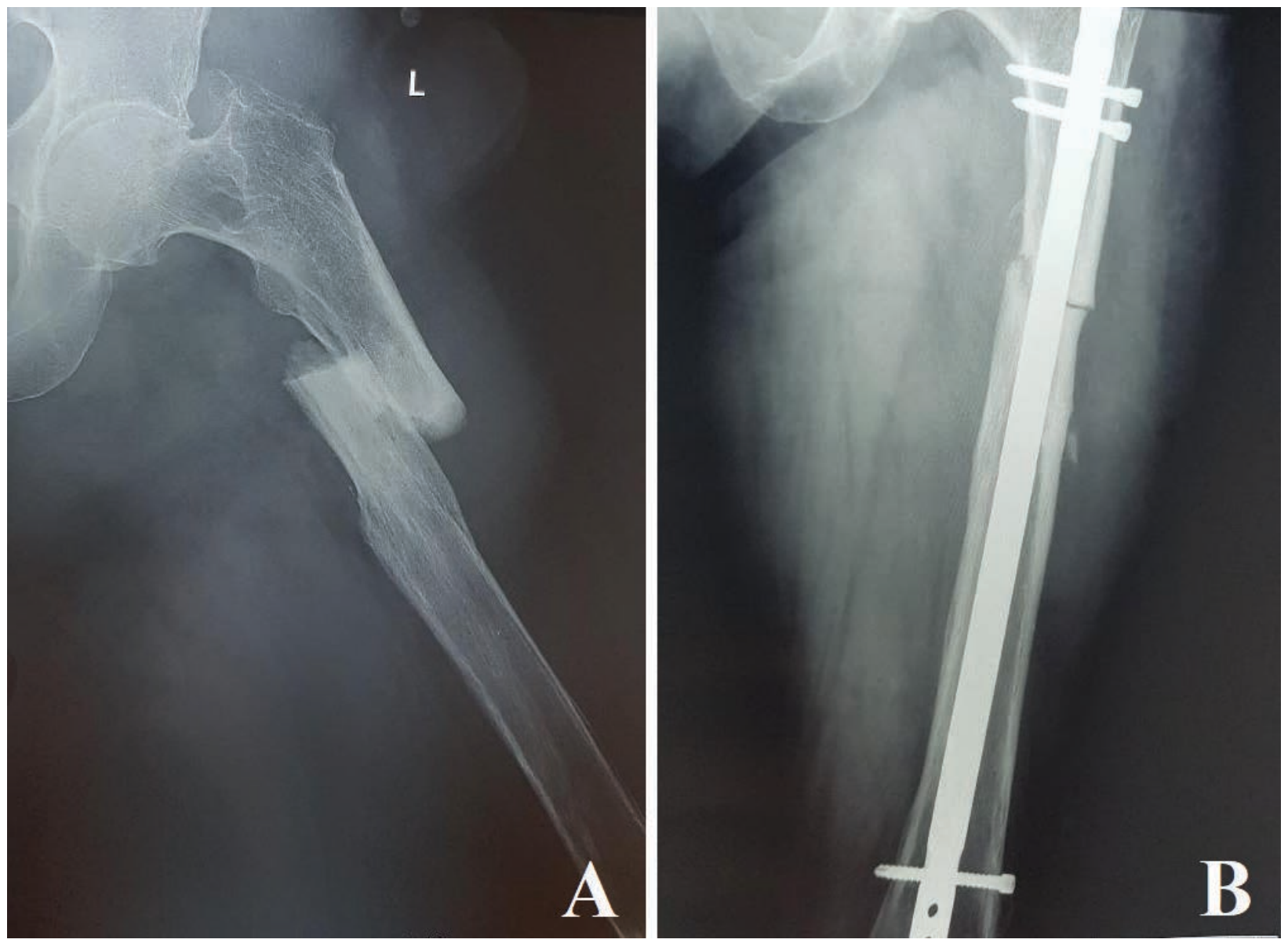

Fig. 1 Left femoral shaft atypical fracture secondary to BPs therapy. (A) Preoperative aspect - X-ray view (anteroposterior incidence), (B) Postoperative aspect - intramedullary nail
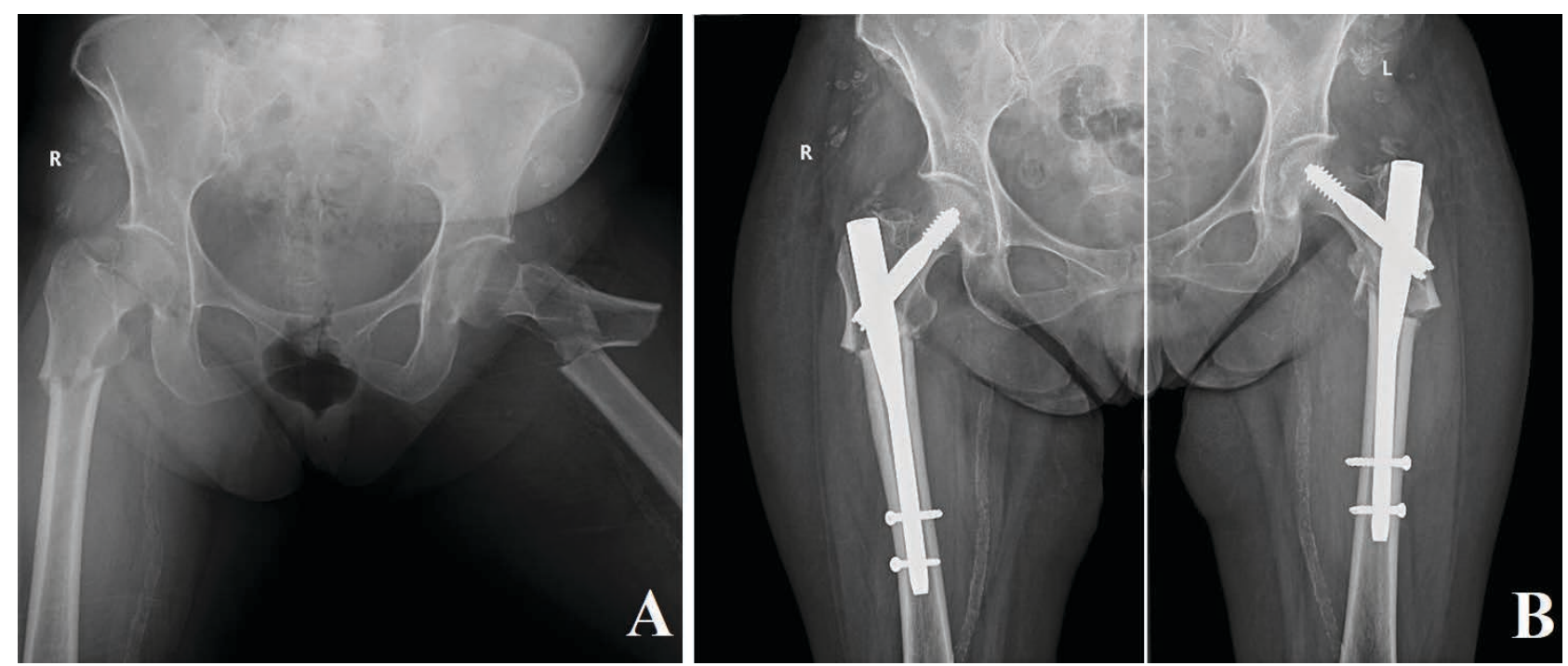

Fig. 2 Bilateral subtrochanteric fracture secondary to BPs therapy. (A) Preoperative aspect-X-ray view (anteroposterior incidence), (B) Postoperative aspect-gamma nail 


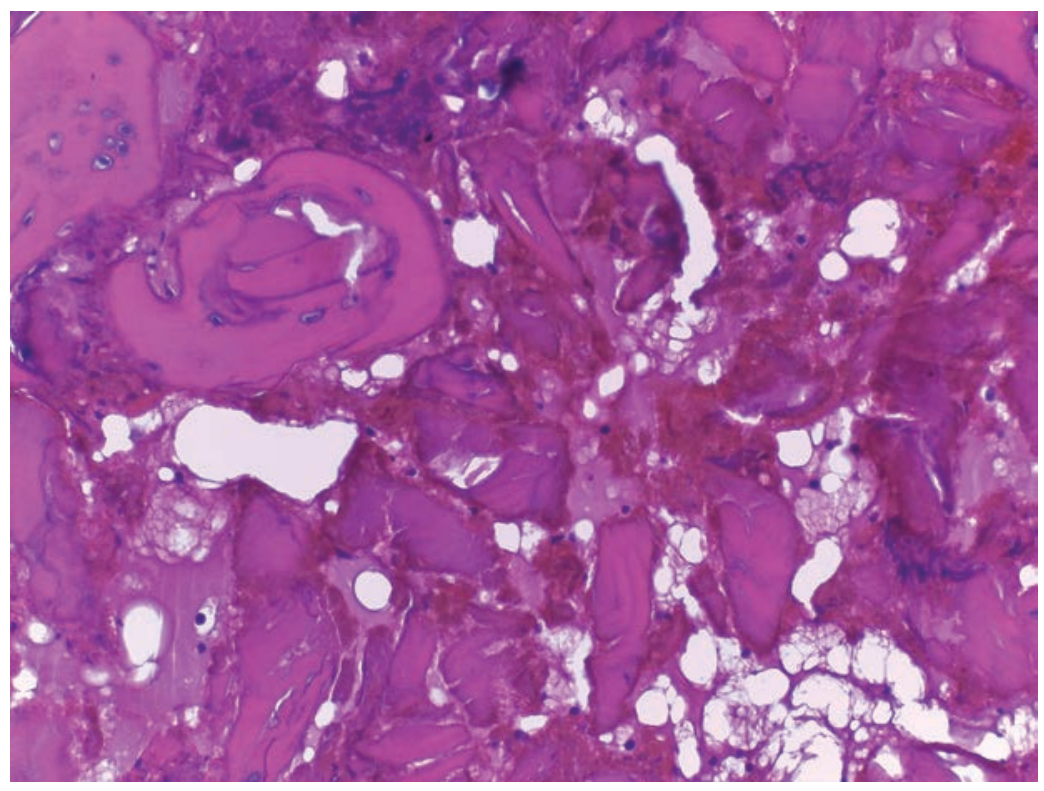

Fig. 3 Cortical bone fragments with extensive aseptic devitalisation (bone lamellae fragmentation and necrosis). The viable bone fragments present basophilic tinctorial and bone resorption in the periphery. Rare chronic inflammatory elements (lymphocytes). Non-necrotic areas with a big quantity of bone, increased trabecular thickness, small and few Haversian canals. Rare osteoclast-like cells in the resorption areas of the viable bone tissue (H.E. ob.20x)

Fig. 4 Mostly devitalized bone tissue, fragmented bone lamellae, whereas in the center - still viable bone material with dystrophic Haversian canal. Rare lymphocytes between bone lamellae (van Gieson ob.10x)

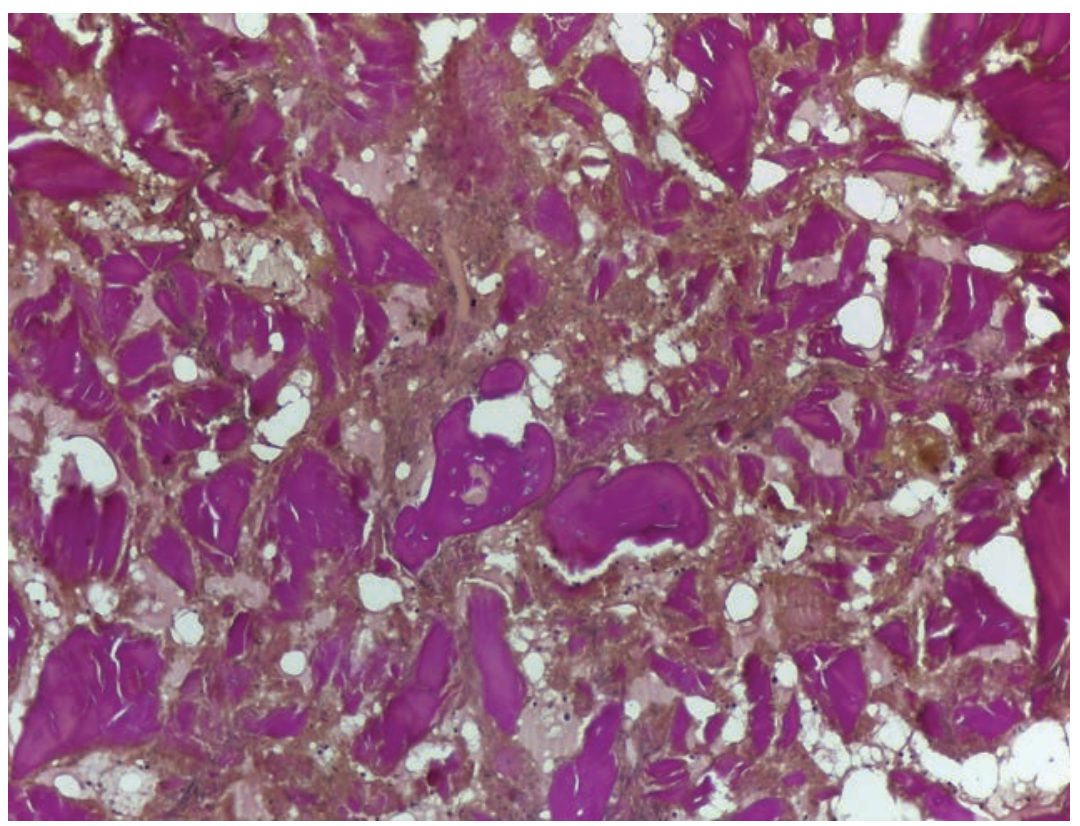

\section{Discussions}

Once published, the results of Odvina study suggesting a causal relationship between longterm treatment with BPs and AFFs, a sustained research activity was initiated regarding this pathology. Consequently, ASBMR published several major and minor criteria for the diagnosis of AFFs, the first ones being published in 2010 and afterwards revised in 2014. For a certain diagnosis of AFFs, at least 4 major criteria are needed, the minor criteria being just orientative [6]. Recognizing an AFF is very important mainly because its postoperative evolution and adjuvant treatment differ from those of a regular fracture. ASBMR recommends the cessation of treatment with $\mathrm{BPs}$, surgical intervention with intramedullary nail in incomplete fractures if the patients have severe pain and conservatory treatment of incomplete fractures in case of absent or moderate pain [7].

In 2010, Dell and Green described an increased incidence of AFFs proportionally with the period of BPs administration. Thus, in case of a 2 year treatment with BPs, the incidence was 2:100000 whereas in case of 8 years of treatment with BPs, the incidence was 78:100000 [8]. Schilcher and Michaelsson revealed a reduction of AFFs with $70 \%$ per year after interrupting BPs therapy [9]. 
Other studies revealed a reduction of bone turnover with subsequent microfractures and decreased bone mechanical resistance in patients with long-term treatment with BPs hence leading to atypical fractures. Similar results were obtained in studies performed in animals with bone metabolism comparable with the human one $[10,11]$.

The influence of BPs therapy on bone metabolism is translated through prolonged time of fracture union and increased incidence of delayed union. The results of our study regarding the mean union were the following: 26 weeks for patients with complete union and 30 weeks when the ones with delayed union were also taken into consideration. Most studies described a mean period of union of AFFs of 6 months [12], although some suggested even 10 months [13]. In addition, an increased incidence of delayed union was registered in female patients with subtrochanteric fractures, the results being very similar to those presented by Lee et al. [12], possibly due to the concentration of muscles in this region, which may lead to higher difficulty in obtaining and maintaining fracture reduction.

\section{Conclusions}

The treatment of AFFs after BPs therapy administration represents a challenge for orthopaedic surgeons regarding both surgical technique and postoperative follow-up.

The positive effect of BPs over osteoporosis should not be neglected, though a follow-up of patients must be performed both from a clinical and biological point of view.

Regarding subtrochanteric fractures secondary to BPs therapy, an increased incidence of postoperative complications and a higher difficulty in obtaining fracture reduction were observed, sometimes even the open reduction being necessary.

\section{Conflict of Interest statements}

Authors state no conflict of interest.

\section{Informed Consent and Human and Animal Rights statements \\ Informed consent has been obtained from}

all individuals included in this study.

\section{Authorization for the use of human subjects}

Ethical approval: The research related to human use complies with all the relevant national regulations, institutional policies, is in accordance with the tenets of the Helsinki Declaration, and has been approved by the authors' institutional review board or equivalent commite

\section{References}

1. Med AJ. Consensus development conference: diagnosis, prophylaxis, and treatment of osteoporosis. Am J Med. 1993; 94:646-50.

2. Nieves JW, Cosman F. Atypical subtrochanteric and femoral shaft fractures and possible association with bisphosphonates. Curr Osteoporos Rep. 2010; 8:34-39.

3. Neviaser AS, Lane JM, Lenart BA, Edobor-Osula F, Lorich DG. Low-energy femoral shaft fractures associated with alendronate use. JOrthop Trauma. 2008; 22:346-350.

4. Bone HG, Schurr W. Intravenous bisphosphonate therapy for osteoporosis: where do we stand?. Curr Osteoporos Rep. 2004; 2:24-30.

5. Odvina CV, Zerwekh JE, Rao DS, Maalouf N, Gottschalk FA, Pak CY. Severely suppressed bone turnover: a potential complication of alendronate therapy. J Clin Endocrinol Metab. 2005; 90:1294-301.

6. Higgins M, Morgan-John S, Badhe S. Simultaneous, bilateral, complete atypical femoral fractures after longterm alendronate use. Journal of Orthopaedics. 2016; 13:401-403.

7. Shane E, Burr D, Ebeling PR et al. Atypical subtrohanteric and diaphyseal femoral fractures: report of a task force of the American Society for Bone and Mineral Research. J Bone Miner Res. 2010; 25:2267-94.

8. Dell R, Greene D, Ott S et al. A Retrospective Analysis of all Atypical Femur Fractures Seen in a Large California HMO from the Years 2007 to 2009. J Bone Miner Res. 2010; (Suppl 1):1201.

9. Schilcher J, Michaelsson K, Aspenberg P. Bisphosphonate use and atypical fractures of the femoral shaft. $\mathrm{N}$ EngI Med. 2011; 364:1728-37.

10. Yamagami Y, Mashiba T, Iwata K et al. Effects of minodronic acid and alendronate on bone remodeling, microdamage accumulation, degree of mineralization and bone mechanical properties in ovariectomized cynomogus monkeys. Bone. 2013; 54:1-7.

11. Allen MR, Burr DB. Changes in vertebral strengh-density and energy absorption-density relationships following bisphosphonate treatment in beagle dogs. Osteoporos Int. 2008; 19:95-99.

12. Lee KJ, Yoo JJ, Oh KJ et al. Surgical outcome of intramedullary nailing in patients with complete atypical femoral fractures: a multicenter retrospective study. Injury, Int J Care Injured. 2017; 48:941-945.

13. Teo BJ, Koh JS, Goh SK et al. Post-operative outcomes of atypical femoral subtrochanteric fracture in patients on bisphosphonates therapy. Bone Joint J. 2014; 96b(5):658-664. 